\title{
Clinical value of anaerobic blood culture: a retrospective analysis of positive patient episodes
}

\author{
Peter A James, Khalid M Al-Shafi
}

\begin{abstract}
Aim-To investigate the clinical value of anaerobic blood culture.

Methods-Blood culture bottles ( $n=25$ 185) submitted for culture over a two year period were reviewed.

Results-The bottles yielded 1992 positive patient episodes, a positive rate of 14.4 / 1000 hospital admissions. Significantly more isolations were obtained from aerobic than from anaerobic bottles. Twelve of the 38 anaerobic episodes were detected in aerobic bottles. Clinical management was influenced in one of 24 patients whose cultures yielded anaerobes from anaerobic bottles only. For a further six patients it was unlikely that the result had any effect on clinical management.

Conclusions-If aerobic bottles were substituted for the anaerobic bottles, detection of positive patient episodes would increase by at least $6 \%$. A higher yield would be achieved by using two aerobic bottles for routine culture and using anaerobic bottles only for patients where anaerobic culture may influence clinical management.

(F Clin Pathol 2000;53:231-233)
\end{abstract}

Microbiology, Gwent

Healthcare NHS Trust,

Royal Gwent Hospital,

Newport, Gwent NP20

2UB, South Wales, UK

P A James

K M Al-Shafi

Correspondence to: Dr James

email: peter.james@

gwent.wales.nhs.uk

Accepted for publication 11 October 1999
Keywords: blood culture; anaerobes; BacT/Alert

Thirty years ago anaerobes accounted for $20-30 \%$ of isolations from blood cultures. ${ }^{1}$ Hence blood culture sets have consisted almost universally of both aerobic and anaerobic culture media. Today, however, largely owing to surgical prophylaxis and improvements in blood culture technology, anaerobes now account for only a small percentage of significant isolates. ${ }^{23}$ We question whether the custom and practice of 30 years ago is appropriate today.

\section{Methods}

Blood culture sets were distributed as follows: a standard aerobic bottle paired with a standard anaerobic bottle (tryptone soy broth based) for routine use, a FAN-aerobic bottle (brain-heart infusion broth based, with activated charcoal) paired with a standard anaerobic bottle for patients on antibiotics or in high dependency units (HDU and ICU), and a single paediatric bottle (brain-heart infusion broth based) for paediatric patients and for patients from whom only a small volume of blood $(<4 \mathrm{ml})$ was obtainable. All (except paediatric) bottles were designed to take $8-10 \mathrm{ml}$ of blood.

On receipt, all bottles (except anaerobic) were vented momentarily in accordance with the manufacturer's recommendations and then incubated at $36^{\circ} \mathrm{C}$ for five days routinely and for 14 days for suspected cases of endocarditis in a standard BacT/Alert 360 system (Organon Teknika). ${ }^{4}$ Positive bottles detected by the continuous monitoring system were examined using standard methods. The significance of isolates was determined through liaison between a medical microbiologist and the clinical team involved in the management of the patient at the time of first detection.

An analysis of data obtained between July 1996 and June 1998 was conducted to determine the range of organisms isolated and the value of each bottle type/combination. Organisms were placed into one of 14 categories (table 1). The patients' clinical notes and

Table 1 Positive patient episodes by bottle type

\begin{tabular}{|c|c|c|c|c|c|c|c|}
\hline \multirow[b]{2}{*}{ Organism group } & \multicolumn{7}{|c|}{ Positive patient episodes by bottle type (rate per 1000 bottles) } \\
\hline & $\begin{array}{l}\text { Both } \\
\text { bottles* }\end{array}$ & $\begin{array}{l}\text { Aerobic } \\
\text { bottle only }\end{array}$ & $\begin{array}{l}\text { FAN-bottle } \\
\text { only }\end{array}$ & $\begin{array}{l}\text { Anaerobic } \\
\text { bottle only }\end{array}$ & $\begin{array}{l}\text { Paediatric } \\
\text { bottle only }\end{array}$ & All & $\begin{array}{l}\text { Percentage } \\
\text { of patients }\end{array}$ \\
\hline Coagulase negative staphylococci & 187 & $227(33.9)$ & $192(40.9)$ & $176(15.5)$ & $145(60.0)$ & 927 & 46.54 \\
\hline Staphylococcus aureus & 98 & $11(1.6)$ & $18(3.8)$ & $15(1.3)$ & $8(3.3)$ & 150 & 7.53 \\
\hline Corynebacteria & 20 & $55(8.2)$ & $13(2.8)$ & $67(5.9)$ & $18(7.4)$ & 173 & 19.18 \\
\hline$\alpha /$ Non-haemolytic streptococci & 28 & $7(1.0)$ & $8(1.7)$ & $21(1.8)$ & $13(5.4)$ & 77 & 3.87 \\
\hline Streptococcus pneumoniae & 39 & $5(0.7)$ & $9(1.9)$ & $10(0.9)$ & $4(1.7)$ & 67 & 3.36 \\
\hline Enterococcus spp & 28 & $4(0.6)$ & $1(0.2)$ & $10(0.9)$ & $3(1.2)$ & 46 & 2.30 \\
\hline$\beta$ Haemolytic streptococci & 14 & $1(0.1)$ & $2(0.4)$ & $3(0.3)$ & $2(0.8)$ & 22 & 1.10 \\
\hline Enterobacteriaceae & 200 & $56(8.4)$ & $55(11.7)$ & $57(5.0)$ & $14(5.8)$ & 382 & 8.68 \\
\hline Pseudomonas spp & 3 & $10(1.5)$ & $11(2.3)$ & $1(0.1)$ & $0(-)$ & 25 & 1.26 \\
\hline Neisseria spp & 1 & $4(0.6)$ & $3(0.6)$ & $0(-)$ & $17(7.0)$ & 25 & 1.26 \\
\hline Haemophilus spp & 3 & $0(-)$ & $0(-)$ & $0(-)$ & $0(-)$ & 3 & 0.15 \\
\hline Anaerobes & 7 & $2(0.3)$ & $2(0.4)$ & $26(2.3)$ & $1(0.4)$ & 38 & 1.91 \\
\hline Yeasts & 9 & $7(1.0)$ & $9(1.9)$ & $1(0.1)$ & $2(0.8)$ & 28 & 1.41 \\
\hline Other & 8 & $9(1.3)$ & $5(1.1)$ & $4(0.4)$ & $3(1.2)$ & 29 & 1.46 \\
\hline Totals & 645 & 398 & 328 & 391 & 230 & 1992 & 100 \\
\hline
\end{tabular}

* "Both bottles" consist of an anaerobic bottle and either a FAN-aerobic or a standard aerobic bottle.

$(\mathrm{n})=$ Isolation rates per 1000 bottles to enable direct comparison with each bottle type.

Unidentifiable organisms were included in "other" along with one each of: Agrobacterium radiobactans, aerobic spore bearer, Chryseomonas luteola, Gemella haemolysans, Gemella morbillorum, Lactobacillus sp, Listeria monocytogenes, and one "mixed culture". Stenotrophomonas spp were included in the "Pseudomonas" category. 


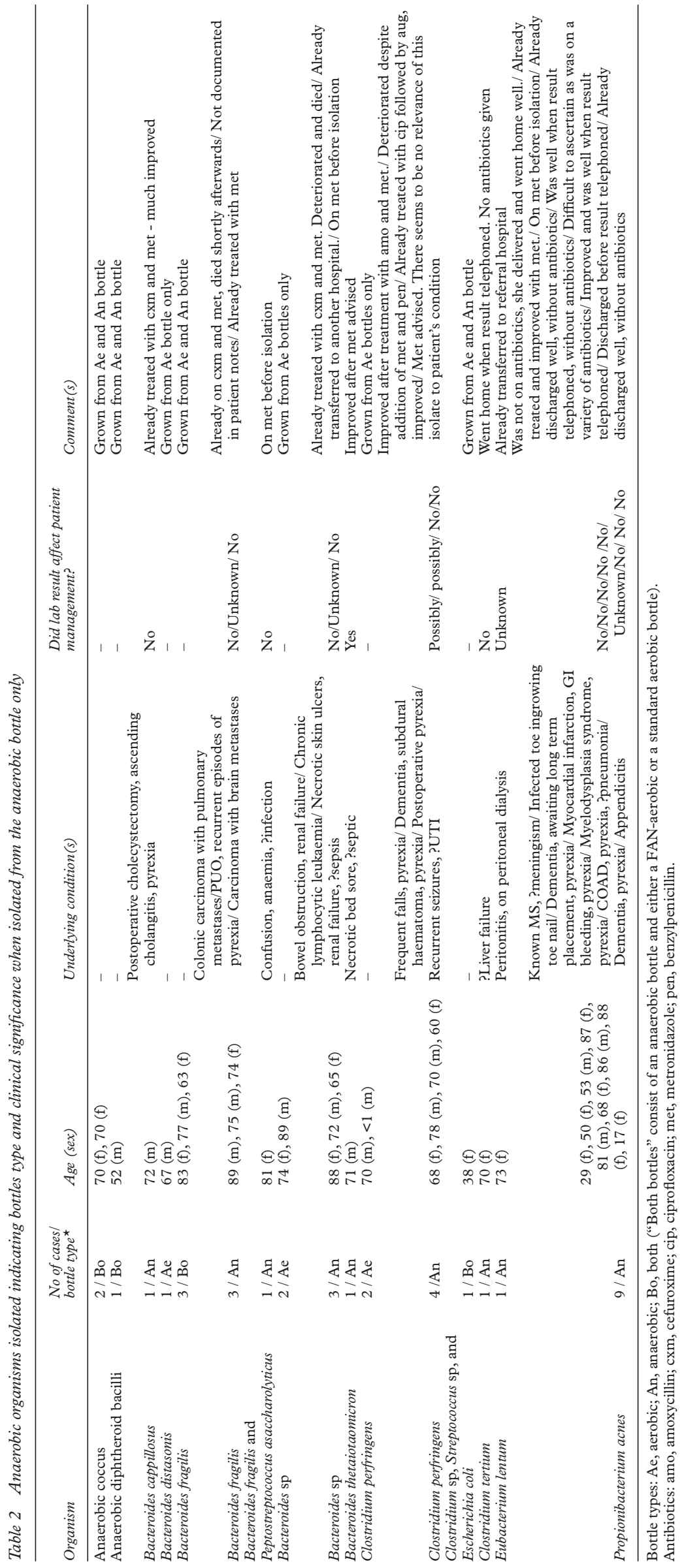

the notes made on laboratory forms from clinical liaison at the time when the culture became positive were studied retrospectively on those from whom anaerobes were grown in the anaerobic bottle only.

\section{Results}

From 25185 blood culture bottles received, there were 1992 positive patient episodes (defined as a single strain isolated from a patient irrespective of the number of positive bottles). The number of hospital admissions for the same period was 137856 patients, (approximately one blood culture set for 10 hospital admissions), with an overall positive patient episode rate of 14.4/1000 hospital admissions. Standard anaerobic bottles $(\mathrm{n}=11377)$ yielded 1115 isolates (9.8\%), compared with standard aerobic bottles $(\mathrm{n}=6698)$ which yielded 835 isolates $(12.5 \%)$, and FAN-aerobic bottles $(\mathrm{n}=4692)$ which yielded 630 isolates (13.4\%). Paediatric bottles $(\mathrm{n}=2418)$ yielded 254 isolates $(10.5 \%)$. Significantly more isolates were obtained from standard aerobic bottles than from standard anaerobic bottles $(p<0.001)$, and there were more isolates from FAN-aerobic bottles than from standard aerobic bottles (NS).

Coagulase negative staphylococci accounted for almost half $(46.5 \%)$ of positive patient episodes. Staphylococcus aureus accounted for $7.5 \%$ and of these $41 \%$ were methicillin resistant (MRSA). Enterobacteriaceae accounted for almost $20 \%$ of isolates, which included four patient episodes of Salmonella spp, all of which were isolated from aerobic bottles. All of the 25 Neisseria spp episodes (24 of which were $N$ meningitidis) were detected in aerobic bottles. Similarly, all four Haemophilus spp grew in both aerobic and anaerobic bottle types. Less than $2 \%$ of positive patient episodes were caused by anaerobes (table 1). More isolations were made from aerobic bottles than from anaerobic bottles for all organism groups except for anaerobes, $\alpha$ haemolytic and non-haemolytic streptococci, and Enterococcus spp (table 1).

Anaerobes were isolated from $37 \mathrm{pa}$ tients (one patient's culture yielded two different anaerobes). In 25 of these, anaerobes were detected from the anaerobic bottles only, while in the remaining 12 the anaerobes were detected in both aerobic and anaerobic bottles (table 2).

\section{Discussion}

The Royal Gwent Hospital is a district general hospital with full range of specialties serving a population of approximately 400000 . Infective episodes caused by anaerobic organisms in this study amounted to $0.28 / 1000$ hospital admissions, though for clinically significant episodes detected by the anaerobic bottle only the rate was no higher than $0.17 / 1000$ hospital admissions. This figure is similar to that reported by Salonen et al..$^{5}$ Of the 
1992 infective episodes detected, only one of the anaerobes isolated from anaerobic bottles alone resulted in a change in the clinical management of the patient - that is, $0.05 \%$ of all infective episodes, though this figure would rise to $0.35 \%$ if we included the six isolates where the effect on patient management could not be established. Though we accept that there are limitations in a retrospective analysis of the clinical impact of blood culture results, we conclude that the anaerobic bottle is of little value for the detection of clinically significant anaerobes that were not isolated in the accompanying aerobic bottle.

The most important factor affecting blood culture isolation is the volume of blood cultured. There is general acceptance that for optimum recovery $20-30 \mathrm{ml}$ of blood should be cultured per venepuncture. ${ }^{4}$ For high volume blood culture systems this means that two bottles a set would be sufficient. The use of a single aerobic bottle would result in at least a $27 \%$ reduction in the detection of positive episodes. This finding is consistent with the fact that for adults each additional millilitre of blood cultured increases the detection rate by up to $3 \%{ }^{4}$ The higher isolation rate in the paediatric bottle is most probably related to the nature of bacteraemia in young children: compared with adults, up to 1000 times more organisms are found per millilitre of blood. ${ }^{4}$

There is much discussion on the appropriateness of different blood culture systems. ${ }^{4678}$ Most investigators agree that, because of the decline in anaerobes and the increase in new and emerging pathogens such as Candida spp, anaerobic culture is less worthwhile now than it was 20 years ago. Others suggest that it is not important to recognise anaerobic bacteraemia microbiologically, as it is easily anticipated clinically and usually treated without waiting for culture confirmation. ${ }^{9}$ Furthermore, antimicrobial susceptibilities of anaerobes are more easily predicted than those for Enterobacteriaceae, non-fermenters, and yeasts. ${ }^{10}$ Therefore it makes sense to optimise blood culture systems in favour of these emerging pathogens.

Propionibacterium acnes, which is nearly always a skin contaminant in our setting (district general hospital), was isolated exclusively from anaerobic bottles, whereas other anaerobes that are more likely to be clinically significant were isolated from both bottle types. Presumably the fact that some anaerobes were detected in the anaerobic bottle only (table 2) merely reflected the sample distribution. Furthermore, in this study, Streptococcus pneumoniae was isolated more often from aerobic bottles in general and far more often, in terms of bottle usage, from FAN-aerobic bottles. We, along with others, ${ }^{9}$ occasionally fail to recover
Str pneumoniae from the standard aerobic bottle owing to autolysis, but this does not appear to be a problem with FAN-aerobic bottles.

Thus the tradition of using a set of blood culture bottles that favours anaerobes to the detriment of aerobic bacteria is being challenged. Our results are consistent with those of other workers in terms of the distribution of isolates between aerobic and anaerobic blood culture bottles. ${ }^{36}$ We suggest that if a second aerobic bottle was substituted for the anaerobic bottles (assuming the same positive rate), there would be an increase in the number of positive patient episodes detected of at least $6 \%$. This has already been predicted, ${ }^{10}$ provided that anaerobic bottles are used for selected patients. We believe that this is a conservative estimate, as there appears to be a further advantage in using two aerobic bottles with different broth systems. It has been reported that not only do FAN-aerobic bottles yield higher isolation rates, particularly for $S$ aureus and enterococci, but also that some organisms seem to have a preference for one bottle type. ${ }^{4}$ It would be possible with the BacT/Alert system to use two bottles with differing broth systems because the standard aerobic bottle is tryptone-soy broth based and the FAN-aerobic bottle is brainheart infusion broth based.

Therefore a higher diagnostic yield would be achieved by using two aerobic bottles, and using anaerobic bottles only for patients where further anaerobic culture may influence patient management, such as patients in high dependency units or those suspected of having deep seated abscesses.

We thank Paul Kennedy for assistance in computer data retrieval.

1 Wilson W, Martin W, Washington II J. Anaerobic bacteraemia. Mayo Clin Proc 1972;47:639-49.

2 Dorsher CW, Rosenblatt JE, Wilson WR, et al. Anaerobic bacteremia: decreasing rate over a 15 year period. Rev Infect Dis 1991;13:633-6.

3 Murray P, Traynor P, Hopson H. Critical assessment of blood culture techniques: analysis of recovery of obligate and facultative anaerobes, strict anaerobic bacteria, and fungi in aerobic and anaerobic blood culture bottles. $\mathcal{F}$ Clin Microbiol 1992;30:1462-8.

4 Reimer LG, Wilson ML, Weinstein MP. Update on detection of bacteremia and fungemia. Clin Microbiol Rev 1997;10:444-65.

5 Salonen J H, Eerola E, Meurman O. Clinical significance and outcome of anaerobic bacteremia. Clin Infect Dis 1998; 26:1413-17.

6 Bannister ER, Woods GL. Evaluation of routine anaerobic blood cultures in the BacT/Alert blood culture system. Am blood cultures in the BacT/Aler

7 Kellogg JA. Selection of a clinically satisfactory blood culture system: the utility of anaerobic media. Clin Microbiol Newslett 1995;17:121-4.

8 Thomas JG. Anaerobic blood cultures: tailoring their selective use. Clin Microbiol Newslett 1995;17:125-8.

9 Sharp SE, McLaughlin JC, Goodman JM, et al. Clinical assessment of anaerobic isolates from blood cultures. Diagn Microbiol Infect Dis 1993;17:19-22.

10 Morris AJ, Wilson ML, Mirrett S, et al. Rationale for selective use of anaerobic blood cultures. F Clin Microbiol 1993; 31:2110-13. 